\title{
Bioinformatics-based study to detect chemical compounds that show potential as treatments for pulmonary thromboembolism
}

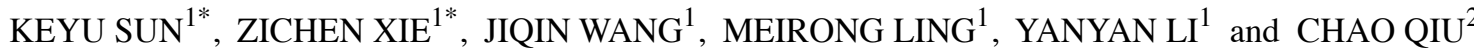 \\ ${ }^{1}$ Emergency Department, Minhang Hospital, Fudan University, Shanghai 201100; \\ ${ }^{2}$ Institute of Biomedical Sciences, Fudan University, Shanghai 200032, P.R. China
}

Received February 9, 2018; Accepted September 27, 2018

DOI: $10.3892 /$ ijmm.2018.3987

\begin{abstract}
The objectives of the present study comprised the recognition of major genes related to pulmonary thromboembolism (PTE) and the evaluation of their functional enrichment levels, in addition to the identification of small chemical molecules that may offer potential for use in PTE treatment. The RNA expression profiling of GSE84738 was obtained from the Gene Expression Omnibus database. Following data preprocessing, the differently expressed genes (DEGs) between the PTE group and the control group were identified using the Linear Models for Microarray package. Subsequently, the protein-protein interaction (PPI) network of these DEGs was examined using the Search Tool for the Retrieval of Interacting Genes/Proteins database, visualized via Cytoscape. The most significantly clustered modules in the network were identified using Multi Contrast Delayed Enhancement, a plugin of Cytoscape. Subsequently, functional enrichment analysis of the DEGs was performed, using the Database for Annotation Visualization and Integrated Discovery tool. Furthermore, the chemical-target interaction networks were investigated using the Comparative Toxicogenomics Database as visualized via Cytoscape. A total
\end{abstract}

Correspondence to: Dr Chao Qiu, Institute of Biomedical Sciences, Fudan University, Fenglin Campus, 138 Shanghai Medical College Road, Xuhui, Shanghai 200032, P.R. China

E-mail: qiuchao@fudan.edu.cn

${ }^{*}$ Contributed equally

Abbreviations: PTE, pulmonary thromboembolism; PE, pulmonary embolism; BNP, brain natriuretic peptide; NT-proBNP, N-terminal pro-BNP; DEGs, differently expressed genes; GEO, Gene Expression Omnibus; PPI, protein-protein interaction; CTD, Comparative Toxicogenomics Database; GEO, Gene Expression Omnibus; GO, Gene Ontology; KEGG, Kyoto Encyclopedia of Genes and Genomes; $\mathrm{BP}$, biological process; CC, cellular component; MF, molecular function; HCM, hypertrophic cardiomyopathy; CNIs, calcineurin inhibitors

Key words: differently expressed genes, protein-protein interaction, chemical-target interactional network of 548 DEGs (262 upregulated and 286 downregulated) were identified in the PTE group, compared with the control group. The upregulated and downregulated genes were enriched in Gene Ontology terms related to inflammation and eye sarcolemma, respectively. Tumor necrosis factor $(T N F)$ and erb-b2 receptor tyrosine kinase 2 (ERBB2) were upregulated genes that ranked higher in the PPI network (47 and 40 degrees, respectively) whereas $C$-JUN was the most downregulated gene (46). Small chemical molecules ethinyl (135), cyclosporine (126), thrombomodulin precursor (113) and tretinoin (111) had $>100$ degrees in the DEG-chemical interaction network. In addition, ethinyl targeted to $T N F$, whereas $T N F$ and $E R B B 2$ were targeted by cyclosporine, and tretinoin was a targeted chemical of $E R B B 2$. Therefore, cyclosporine, ethinyl, and tretinoin may be potential targets for PTE treatment.

\section{Introduction}

Acute pulmonary thromboembolism (PTE), the obstruction by a thrombus in the pulmonary artery or its branches, is the most common form of pulmonary embolism (PE) (1). The common pathology of PTE comprises hemodynamic instability, hypoxia, and pulmonary hypertension, which may cause heart failure with hypotension $(2,3)$. PTE is reported to be a complex disease caused by a variety of factors, including the vascular microenvironment and vascular cell dysfunction $(4,5)$. During an episode of PTE, a thrombus trapped in the pulmonary blood vessels injures the vascular endothelium, causing the unregulated release of pro-inflammatory mediators $(2,6)$. Pulmonary vascular remodeling caused by repeated pulmonary vascular injury may lead to secondary pulmonary hypertension (7), a major clinical consequence of PTE, which indicates that endothelial injury induced by PTE may be key in the pathophysiological consequences of PTE (8). PTE is a common disease, which is a contributing factor to the global non-communicable disease burden $(8,9)$.

Previous studies have identified brain natriuretic peptide (BNP) and N-terminal pro-BNP (NT-proBNP) in the blood as biomarkers for predicting echocardiographic right ventricular dysfunction in patients with acute PTE $(10,11)$. Furthermore, it is reported that troponin I, D-dimer, and plasma tenascin-C are positively correlated with the occurrence of PTE, whereas the erythrocyte sedimentation rate is downregulated in patients with PTE (12). Tang et al identified interleukin 8 (IL-8), 
tumor necrosis factor- $\alpha(T N F-\alpha)$, and C-X-C motif chemokine ligand 5 as important factors in PTE in rabbits (13). In addition, it was revealed that these important genes are associated with cardiovascular disease, pulmonary disease, immune disease, and inflammation (13). However, small chemical molecules targeting differently expressed genes (DEGs) between the PTE group and the control group did not screen out. The use of small chemical molecules that target important disease-related genes has become an effective strategy for disease treatment $(14,15)$.

In the present study, the GSE84738 dataset was obtained from the Gene Expression Omnibus (GEO) database. Following data preprocessing, the DEGs between a PTE group and control group were identified. Subsequently, the protein-protein interaction (PPI) network of these DEGs was visualized using Cytoscape, and the most significantly clustered modules in the network were analyzed. Subsequently, functional enrichment analysis of the DEGs was performed. Furthermore, the chemical-target interaction networks were investigated using the Comparative Toxicogenomics Database (CTD), visualized via Cytoscape. The present study identified the important genes and their functional enrichment in relation to the occurrence of PTE, and identified small chemical molecules potentially useful for PTE treatment. These findings may provide an important basis to detect mechanisms underlying PTE and potential treatment methods for PTE.

\section{Materials and methods}

Data sources. The RNA expression profiling dataset of the European rabbit (Oryctolagus cuniculus) was obtained from the GEO database in the National Center for Biotechnology Information (http://www.ncbi.nlm.nih.gov/geo/; accession no. GSE84738) (13). The dataset included eight samples (four control samples and four PTE samples), collected from rabbit pulmonary artery tissue. All samples were assessed, based on the GPL13288 Agilent-020908 Oryctolagus cuniculus (rabbit) Oligo Microarray platform. The use of animals was approved by the Animal Ethics Committee of Affiliated Hospital of Nantong University (Nantong, China).

Screening of DEGs. The original file was downloaded and annotated. In cases where one gene was detected by multiple probes, the mean value of the probes was used to represent the expression level. The gene expression matrix was separated into a control group and a PTE group, and the DEGs between the two groups were screened according to the Linear Models for Microarray package (version 3.30.3, www.bioconductor. org/packages/release/bioc/html/limma.html) in R software. The P-values of these DEGs were calculated and adjusted using an unpaired t-test and Benjamini-Hochberg method, respectively, at a significance level of $\mathrm{P}<0.05$. Subsequently, the heatmap in $\mathrm{R}$ package, which is used for drawing heatmaps of gene expression, was utilized to plot the heat map of DEGs (16). Clustering between the different samples and different genes was performed using Pearson's and Spearman's correlation coefficients, respectively.

Analysis of the PPI network. The PPIs of the DEGs between the control group and PTE group were extracted from the
Search Tool for the Retrieval of Interacting Genes/Proteins database (version 10.0, http://www.string-db.org/) with default parameters (species: Oryctolagus cuniculus) (17). Those PPIs meeting the combined score requirement of $>0.4$ were used to construct the PPI network, which was then visualized using Cytoscape (version 3.2.0, http://cytoscape.org/) (18). In the PPI network, each node represents a protein, and each edge between two nodes represents an interaction between these two proteins. The degree is defined as the number of proteins interacting with the node.

Furthermore, the degree centrality, a network topology index, was used to analyze the scores of nodes in the network. The nodes with higher degrees are considered to be important in the PPI network, and may be the key nodes.

Subnet module analysis. Genes often have a regulatory role by interacting with other cellular components. Proteins produced by genes in the same module tend to have the same function or similar functions, and where they act as a module they may have the same biological role. The most significantly clustered modules in the network were analyzed using the Multi Contrast Delayed Enhancement plugin of Cytoscape (19).

GO and Kyoto Encyclopedia of Genes and Genomes (KEGG) pathway analysis of regulatory network modules. In order to examine the gene function and pathways related to the DEGs, the Database for Annotation Visualization and Integrated Discovery (DAVID) online tool (version 6.8, https://david-d. ncifcrf.gov/) (20) was used to perform the Gene Ontology (GO) functional and pathway enrichment analyses of DEGs based on the GO database (20) and KEGG pathway database (21). Only function terms with an adjusted P-value of $<0.05$ and count $\geq 2$ were considered as significant.

Analysis of disease-related chemistry small molecule-target gene interaction network. The CTD, a publicly available database (http://ctdbase.org/), provides manually curated information on chemical-disease, gene-disease associations and chemical-gene/protein interactions. Based on PE-related chemical-gene interaction data provided by the CTD (22), the chemical-gene interaction association pairs were visualized via Cytoscape. Chemicals interacting with a higher number of DEGs may be more important in this disease.

\section{Results}

Identification of DEGs. A total of 548 (262 upregulated and 286 downregulated DEGs) were identified in the PTE group, compared with the control group. The results of the hierarchical clustering heat map of DEGs showed that these DEGs were clearly distinguishable between the different sample types (Fig. 1).

Functional enrichment analysis. The upregulated DEGs were significantly enriched in $27 \mathrm{KEGG}$ pathways, $51 \mathrm{GO}$ biological processes (BPs), $27 \mathrm{GO}$ cellular components (CCs) and $10 \mathrm{GO}$ molecular functions (MFs). The downregulated genes were significantly enriched in 11 KEGG pathways, 15 GO BPs, 14 GO CCs, and 11 GO MFs. The enriched results are presented in Table I. The upregulated DEGs were 
Table I. Top 10 Kyoto Encyclopedia of Genes and Genomes pathways enriched by differentially expressed genes.

\begin{tabular}{|c|c|c|c|}
\hline Pathway ID & Pathway name & Count & P-value \\
\hline \multicolumn{4}{|l|}{ Upregulated } \\
\hline ocu04145 & Phagosome & 15 & $5.51 \mathrm{E}-09$ \\
\hline ocu04640 & Hematopoietic cell lineage & 10 & 4.39E-07 \\
\hline ocu05323 & Rheumatoid arthritis & 10 & $1.99 \mathrm{E}-06$ \\
\hline ocu05140 & Leishmaniasis & 9 & 4.03E-06 \\
\hline ocu05162 & Measles & 9 & $1.80 \mathrm{E}-04$ \\
\hline ocu05142 & Chagas disease (American trypanosomiasis) & 8 & 3.94E-04 \\
\hline ocu04620 & Toll-like receptor signaling pathway & 7 & $8.54 \mathrm{E}-04$ \\
\hline ocu05133 & Pertussis & 6 & $2.08 \mathrm{E}-03$ \\
\hline ocu05146 & Amoebiasis & 7 & $2.30 \mathrm{E}-03$ \\
\hline ocu05144 & Malaria & 5 & $3.61 \mathrm{E}-03$ \\
\hline \multicolumn{4}{|c|}{ Downregulated } \\
\hline ocu05412 & Arrhythmogenic right ventricular cardiomyopathy & 7 & $5.12 \mathrm{E}-05$ \\
\hline ocu04020 & Calcium signaling pathway & 9 & $2.26 \mathrm{E}-04$ \\
\hline ocu05410 & Hypertrophic cardiomyopathy & 6 & $1.24 \mathrm{E}-03$ \\
\hline ocu05414 & Dilated cardiomyopathy & 6 & $1.66 \mathrm{E}-03$ \\
\hline ocu04080 & Neuroactive ligand-receptor interaction & 9 & $2.90 \mathrm{E}-03$ \\
\hline ocu04022 & cGMP-PKG signaling pathway & 7 & 4.86E-03 \\
\hline ocu04261 & Adrenergic signaling in cardiomyocytes & 6 & $1.11 \mathrm{E}-02$ \\
\hline ocu04066 & HIF-1 signaling pathway & 5 & $1.20 \mathrm{E}-02$ \\
\hline ocu05416 & Viral myocarditis & 4 & $2.74 \mathrm{E}-02$ \\
\hline ocu04151 & PI3K-Akt signaling pathway & 8 & $2.75 \mathrm{E}-02$ \\
\hline
\end{tabular}

cGMP-PKG, cyclic guanosine monophosphate-dependent protein kinase G; HIF-1, hypoxia-inducible factor-1; PI3K, phosphoinositide-3-kinase.

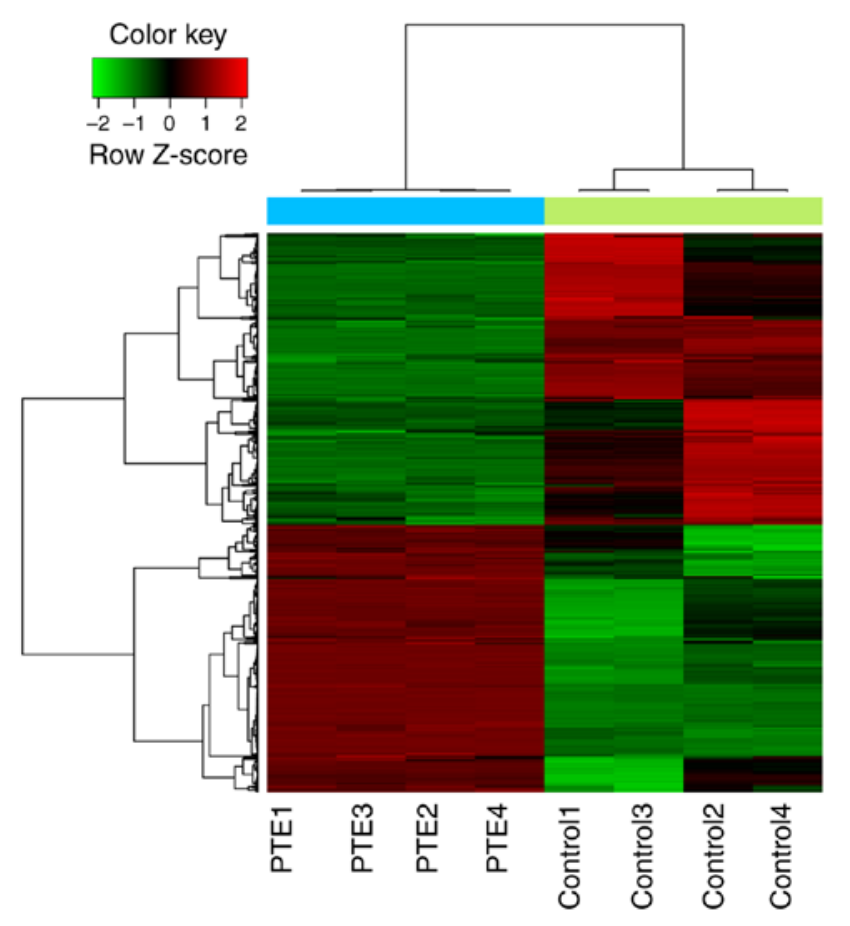

Figure 1. Clustered heat map of differentially expressed genes in the PTE group compared with the control group. The abscissa represents different samples, and the ordinate represents different genes. The red boxes indicate upregulated genes, and the green boxes indicate downregulated genes. Clustering between different samples and different genes were performed based on Pearson's and Spearman's correlation coefficients, respectively. PTE, pulmonary thromboembolism. largely enriched in the phagosome $(\mathrm{P}=5.51 \mathrm{E}-09)$ and hematopoietic $(\mathrm{P}=4.39 \mathrm{E}-07$, e.g. $T N F)$ cell lineages in the KEGG pathways, and the downregulated DEGs were enriched in the arrhythmogenic right ventricular cardiomyopathy $(\mathrm{P}=5.12 \mathrm{E}-05)$ and calcium signaling $(\mathrm{P}=2.26 \mathrm{E}-04)$ pathways in the KEGG pathways (Table I). Interleukin-6 production $(\mathrm{P}=4.02 \mathrm{E}-07$, e.g. TNF $)$ in GO BP, external side of plasma membrane ( $\mathrm{P}=1.46 \mathrm{E}-05$, e.g. $T N F)$, NADPH oxidase complex $(\mathrm{P}=2.08 \mathrm{E}-05)$ in GO CC, and low-density lipoprotein particle binding $(\mathrm{P}=6.06 \mathrm{E}-06)$ in $\mathrm{MF}$ were the upregulated genes, and were enriched among the abovementioned GO terms (Fig. 2A). The downregulated genes were largely enriched in lens development in camera-type eye $(\mathrm{P}=2.40 \mathrm{E}-05)$, sarcolemma $(\mathrm{P}=5.93 \mathrm{E}-05)$, and structural constituent of eye lens $(\mathrm{P}=1.01 \mathrm{E}-08)$ (Fig. 2B).

PPI network and module analysis. A total of 342 nodes and 1,128 interactional pairs were included in the PPI network. Among them, 10 proteins had $>25$ degrees (Fig. 3). TNF and $E R B B 2$ were the upregulated genes with the highest degree in the PPI network, with 47 and 40 degrees respectively. $C$-JUN was the downregulated gene with the highest degree of 46 . Furthermore, $C-J U N$ was an interacting protein of $T N F$ and ERBB2.

In total, three subnet modules (A-C) were identified with a score $>4$. Module A (score $=6.769$ ) included 14 nodes (e.g. actin $\beta$ ) and 44 interactional pairs (Fig. 4A). Module B (score $=6)$ had seven nodes (e.g. potassium voltage-gated 

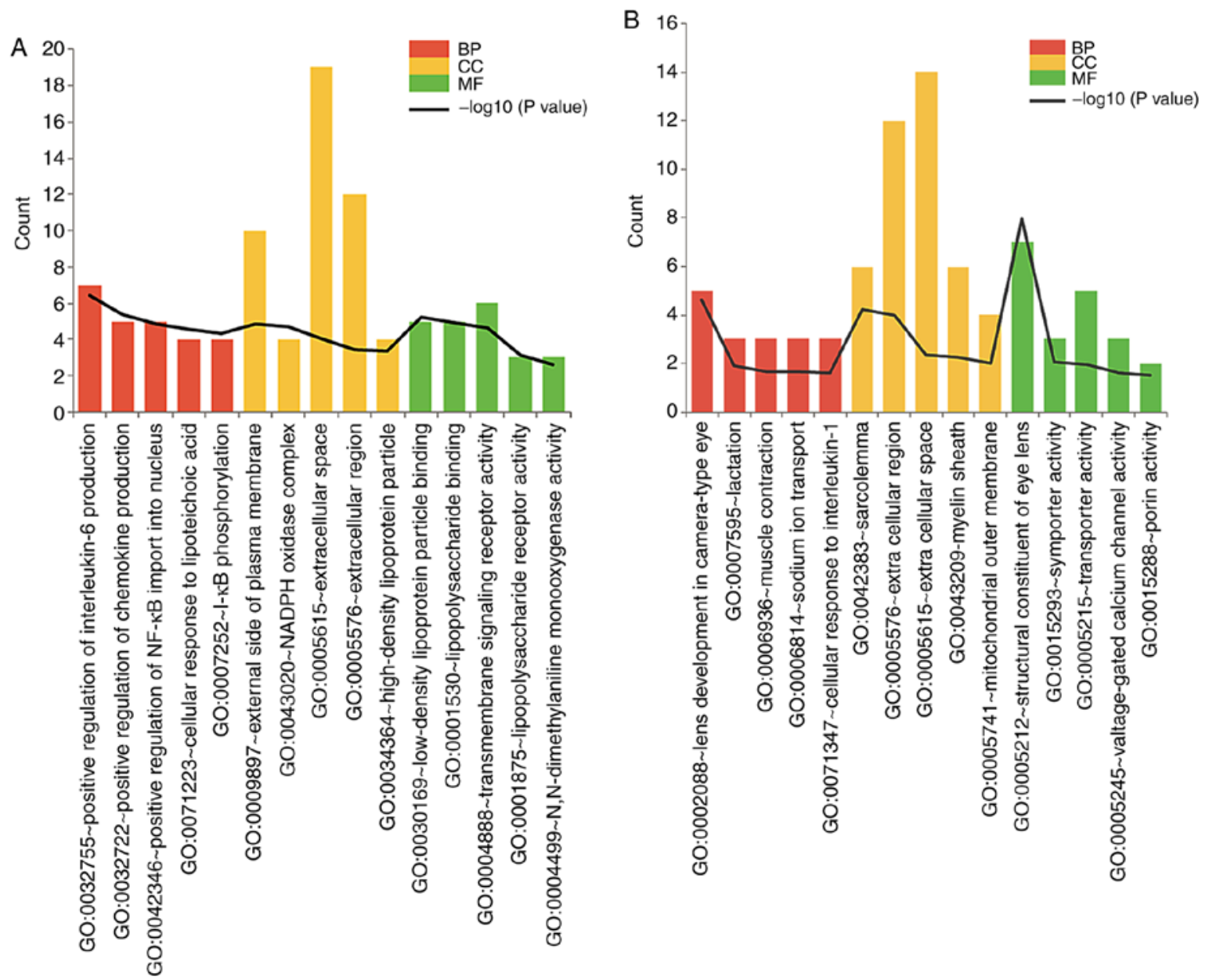

Figure 2. Top GO terms enriched in DEGs. Top 5 GO terms enriched in (A) upregulated and (B) downregulated DEGs. Red, yellow, and green boxes represent GO BP, CC, and MF, respectively. GO, gene ontology; DEGs, differentially expressed genes; BP, biological process; CC, cellular component; MF, molecular function.

channel subfamily B member 2) and 18 interactional pairs (Fig. 4B). Module $\mathrm{C}$ (score $=4.435)$ included 24 nodes (e.g. $T N F$ and $C-J U N)$ and 51 interactional pairs (Fig. 4C). Genes in module A were most enriched in cardiac muscle contraction $(\mathrm{P}=3.97 \mathrm{E}-03)$, hypertrophic cardiomyopathy $(\mathrm{P}=4.79 \mathrm{E}-03)$ and dilated cardiomyopathy $(\mathrm{P}=5.45 \mathrm{E}-03)$. Genes in module $\mathrm{B}$ were most enriched in the oxytocin signaling pathway $(\mathrm{P}=1.33 \mathrm{E}-03)$, whereas biosynthesis of antibiotics in the KEGG pathways $(\mathrm{P}=1.61 \mathrm{E}-03)$ was enriched in module $\mathrm{C}$ genes.

PTE-related chemical-small molecule-target gene interaction network. In the CTD database, 50,719 PE-related chemical-target interactions, including 1,537 DEG-chemical interactions, were detected (Fig. 5). A total of 1,537 interactional pairs, 127 upregulated DEGs, 105 downregulated DEGs, and 117 chemicals, including four with a degree of $>100$ [ethinyl (135), cyclosporine (126), thrombomodulin precursor (THBD) (113) and tretinoin (111)], were found in the DEG-chemical interaction network. Among them, ethinyl targeted $T N F$, whereas $E R B B 2$ were targeted by cyclosporine. In addition to $T H B D$ being an upregulated gene, it was an important chemical interacting with ethinyl, cyclosporine and tretinoin. The chemical tretinoin targeted ERRB2 (Table II).

\section{Discussion}

The findings of the present study demonstrated that $T N F$ and $E R B B 2$ were upregulated, and $C-J U N$ was the main downregulated gene in the PTE group. Additionally, ethinyl, cyclosporine, and tretinoin may have significant effects in the treatment of PTE. Among them, TNF was targeted by ethinyl and cyclosporine, $E R B B 2$ was targeted by cyclosporine and tretinoin, and $C$-JUN was targeted by $T N F$ and $E R B B 2$.

$T N F$ is a multifunctional pro-inflammatory cytokine produced by macrophages. Previous studies have indicated that inflammation is associated with the occurrence of PTE (23). Due to stimulation caused by hypoxia and trauma during PTE, inflammation may trigger pulmonary platelet activation and endothelial dysfunction (24). Additionally, systemic inflammatory and leukocytosis responses are prognostic factors for mortality following 30 days of PTE (25). In addition, pro-inflammatory factors, including $T N F$ and $I L-6$, are significantly correlated with PTE mortality rate $(26,27)$. Consistent with previous studies, the results of the present study suggested that, compared with the control group, $T N F$ was upregulated in the PTE group, and that it was mainly enriched in the hematopoietic cell lineage, positive regulation of interleukin-6 production, and external side of the plasma membrane. 


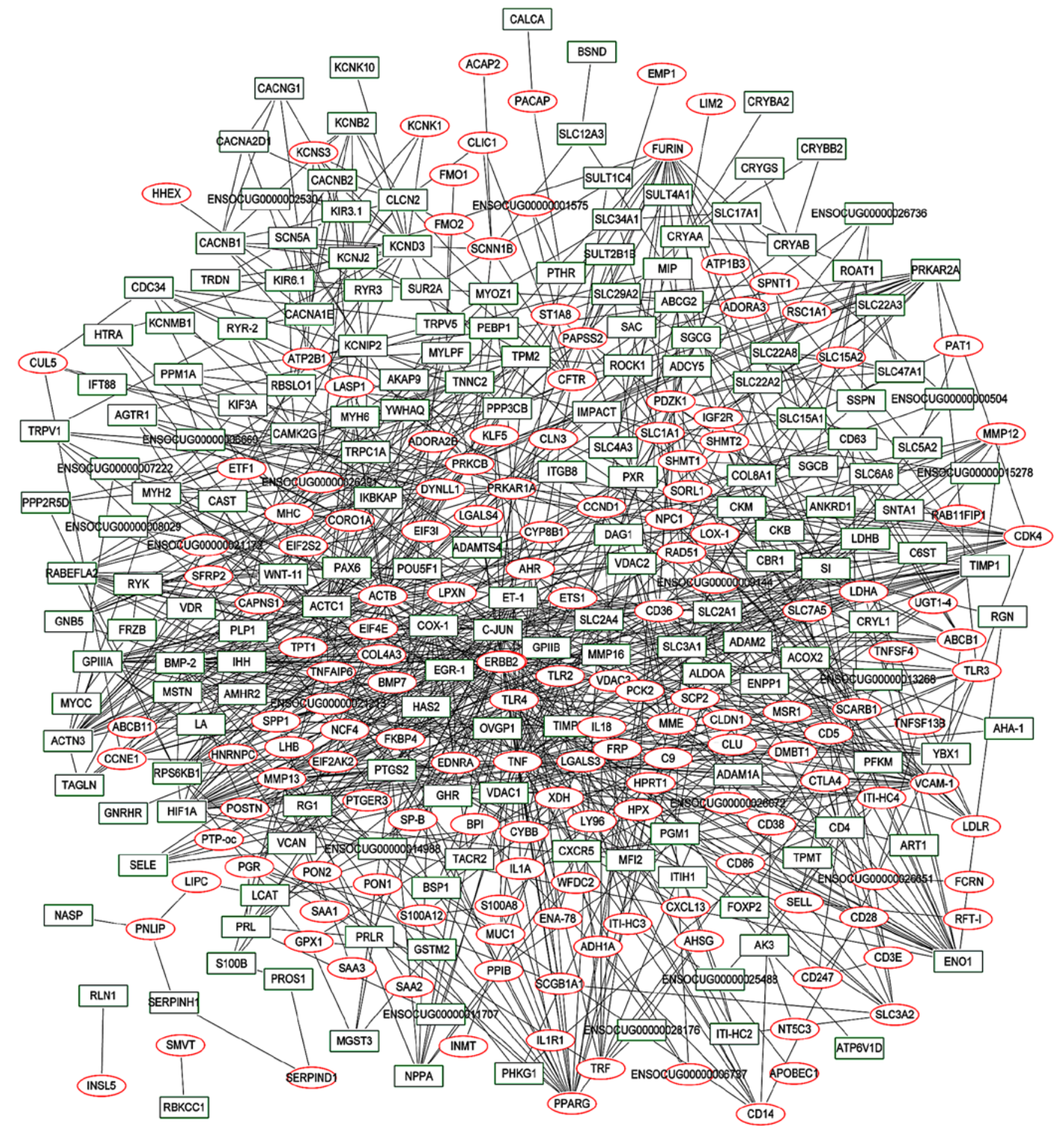

Figure 3. Protein-protein interaction network of differentially expressed genes. Red circles and green squares represent the upregulated and downregulated genes, respectively. Lines indicate protein-protein interaction.

Furthermore, it was targeted by small chemical molecules, including ethinyl and cyclosporine. Ethinyl is a contraceptive, although oral ethinyl increases the risk of venous thromboembolisms including PTE $(28,29)$. Cyclosporine is a calcineurin inhibitor (CNI), which is beneficial for reducing the incidence of complications during homologous transplantation, including vascular toxicity (30). CNIs induce proinflammatory cytokine production and endothelial activation in the isolated mouse aorta and vascular smooth muscle cells (31). Cyclosporine also increases the mRNA levels of proinflamma- tory cytokines, including $T N F-\alpha, I L-6, \mathrm{C}-\mathrm{C}$ motif chemokine ligand 5 (CCL5), and CCL2, which are involved in vascular injury (31). Consistent with previous studies, the results of the present study indicated that TNF was targeted by cyclosporine. Cyclosporine promotes inflammation-induced monocyte adhesion to human intestinal endothelial cells (32). Therefore, ethinyl and cyclosporine may increase the incidence of PTE via targeting $T N F$ and promoting the onset of inflammation.

Cyclosporine is also targeted by $E R B B 2$, which is a member of the epidermal growth factor receptor family. 
A

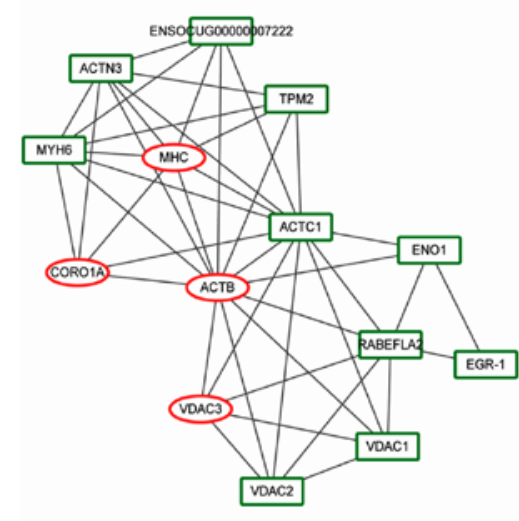

B

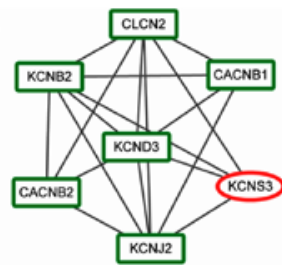

C

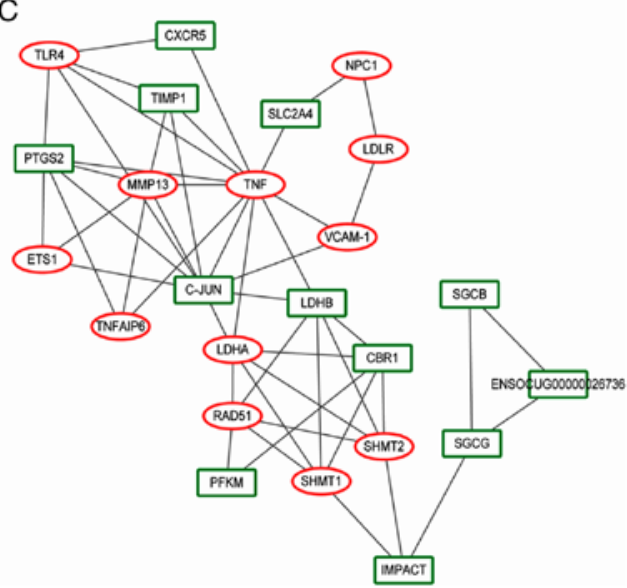

Figure 4. Results of subnet analysis of the PPI network. (A) Module A, (B) module B, and (C) module C of the PPI network are presented. Red circles and green squares represent the upregulated and downregulated genes, respectively. The lines indicate PPI. PPI, protein-protein interaction.

Table II. Top five Kyoto Encyclopedia of Genes and Genomes pathways enriched by differentially expressed genes in the top 10 degrees and modules.

\begin{tabular}{|c|c|c|c|c|}
\hline Pathway ID & Pathway name & Count & P-value & Genes \\
\hline \multicolumn{5}{|l|}{ Degree top 10} \\
\hline ocu05205 & Proteoglycans in cancer & 5 & 7.67E-06 & ACTB, HIF1A, TNF, ERBB2, TLR4 \\
\hline ocu05140 & Leishmaniasis & 3 & $1.53 \mathrm{E}-03$ & TNF, PTGS2, TLR4 \\
\hline ocu04064 & NF- $\kappa \mathrm{B}$ signaling pathway & 3 & $1.69 \mathrm{E}-03$ & TNF, PTGS2, TLR4 \\
\hline ocu05410 & Hypertrophic cardiomyopathy & 3 & $2.04 \mathrm{E}-03$ & ACTB, ACTC1, TNF \\
\hline ocu05414 & Dilated cardiomyopathy & 3 & $2.33 \mathrm{E}-03$ & ACTB, ACTC1, TNF \\
\hline \multicolumn{5}{|l|}{ Module A } \\
\hline ocu04260 & Cardiac muscle contraction & 3 & 3.97E-03 & ACTC1, MYH6, TPM2 \\
\hline ocu05410 & Hypertrophic cardiomyopathy & 3 & 4.79E-03 & АСТВ, АCTC1, ТРM2 \\
\hline ocu05414 & Dilated cardiomyopathy & 3 & $5.45 \mathrm{E}-03$ & АCTB, АCTC1, ТPM2 \\
\hline ocu04530 & Tight junction & 3 & $1.08 \mathrm{E}-02$ & ACTB, MYH6, ACTN3 \\
\hline ocu04261 & Adrenergic signaling in cardiomyocytes & 3 & $1.29 \mathrm{E}-02$ & АCTC1, MYH6, ТPM2 \\
\hline \multicolumn{5}{|l|}{ Module B } \\
\hline ocu04921 & Oxytocin signaling pathway & 3 & $1.33 \mathrm{E}-03$ & $\mathrm{CACNB} 1, \mathrm{CACNB} 2, \mathrm{KCNJ} 2$ \\
\hline ocu05412 & $\begin{array}{l}\text { Arrhythmogenic right ventricular } \\
\text { cardiomyopathy }\end{array}$ & 2 & $2.94 \mathrm{E}-02$ & CACNB1, CACNB2 \\
\hline ocu04260 & Cardiac muscle contraction & 2 & $3.22 \mathrm{E}-02$ & CACNB1, CACNB2 \\
\hline ocu05410 & Hypertrophic cardiomyopathy & 2 & $3.53 \mathrm{E}-02$ & CACNB1, CACNB2 \\
\hline ocu05414 & Dilated cardiomyopathy & 2 & 3.77E-02 & CACNB1, CACNB2 \\
\hline \multicolumn{5}{|l|}{ Module C } \\
\hline ocu01130 & Biosynthesis of antibiotics & 5 & $1.61 \mathrm{E}-03$ & SHMT1,LDHB, LDHA, SHMT2, PFKM \\
\hline ocu00010 & Glycolysis/gluconeogenesis & 3 & $1.07 \mathrm{E}-02$ & LDHB, LDHA, PFKM \\
\hline ocu01230 & Biosynthesis of amino acids & 3 & $1.25 \mathrm{E}-02$ & SHMT1, SHMT2, PFKM \\
\hline ocu05140 & Leishmaniasis & 3 & $1.29 \mathrm{E}-02$ & TNF, PTGS2, TLR4 \\
\hline ocu04064 & NF-кB signaling pathway & 3 & $1.42 \mathrm{E}-02$ & TNF, PTGS2, TLR4 \\
\hline
\end{tabular}

$\mathrm{NF}-\kappa \mathrm{B}$, nuclear factor- $\kappa \mathrm{B}$. 


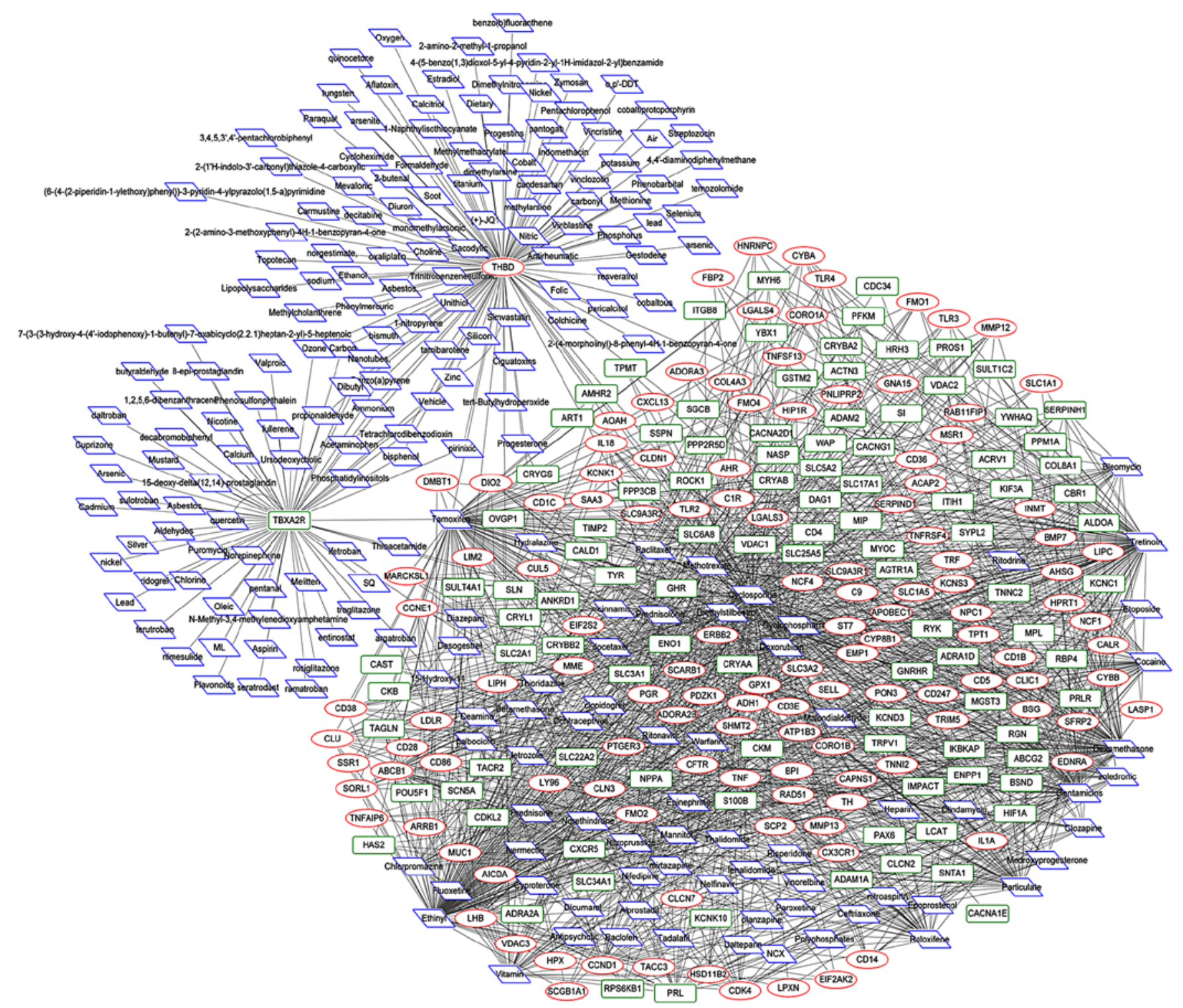

Figure 5. Chemical-target interactional network. The blue quadrilaterals, red circles, and green squares represent the chemical small molecules, the upregulated genes, and the downregulated genes, respectively. Lines indicate protein-protein interaction.

$E R B B 2$ is increased in patients with cancer, including breast and ovarian cancer (33). In breast cancer, ERBB2 is important in regulating oncogenic microRNA expression (34). ERBB2 is also a potent independent predictor of the metastatic potential of breast cancer cells (35). However, few studies appear to have investigated the role of ERBB2 in PTE. It is reported that breast cancer is closely associated with the development of vascular emboli (36). In the present study, $E R B B 2$ was the important upregulated gene in the PTE group, as it had a higher degree in the PPI network, and it was a target of the small chemical molecules, cyclosporine and tretinoin. Tretinoin, a metabolite of retinol, can repair lung tissue in pulmonary emphysema model rats (37). The role of tretinoin in promoting alveolar regeneration involves the regulation of vascular endothelial growth factor (VEGF), VEGF receptor 2, and matrix metalloproteinase 1 (38). Therefore, tretinoin may reduce the degree of lung injury in PTE by downregulating ERBB2. Cyclosporine may contribute to the occurrence of PTE via targeting ERBB2 and $T N F$, thereby regulating the expression of $C-J U N$.
In humans, C-JUN is a protein encoded by JUN. C-JUN in combination with $\mathrm{C}-\mathrm{FOS}$, forms the early response transcription factor, activator protein 1 (39). In addition, C-JUN cooperates with nuclear factor- $\kappa \mathrm{B}$ to prevent apoptosis induced by TNF- $\alpha$. C-JUN can also protect hepatocytes from apoptosis, as hepatocytes lacking C-JUN show increased sensitivity to TNF- $\alpha$-induced apoptosis (40). However, the role of C-JUN in PTE has not been reported. As stated above, pro-inflammatory factors, including $T N F$ and $I L-6$, show a significant correlation with PTE mortality rate. In addition, $\beta 4$ integrin forms a complex with ERBB2 and enhances activation of the transcription factors signal transducer and activator of transcription 3 and C-JUN (41). C-JUN is also necessary for ERBB2-mediated hyperproliferation. Therefore, $C-J U N$ may regulate $T N F$ and $E R B B 2$.

However, the results of the present study have not been experimentally validated, which is a limiting factor that requires resolution in the future. In addition, the regulation of small chemical molecules by PTE-related gene expression 
was not investigated. The sample size used in the present study was also small (four control and four PTE pulmonary artery samples).

In conclusion, the findings of the present study indicate that small chemical molecules, cyclosporine and ethinyl, may trigger PTE by regulating the expression of $T N F$ and $E R B B 2$. Furthermore, tretinoin may delay the progression of PTE via targeting ERBB2. Taken together, cyclosporine, ethinyl, and tretinoin may be promising potential targets for PTE treatment.

\section{Acknowledgements}

Not applicable.

\section{Funding}

The present study was supported by the Shanghai Medical Key Subject Construction Project (grant no. ZK2015B15), the Shanghai Weak Discipline Construction Plan of Shanghai Municipal Commission of Health and Family Planning (grant no. 2016ZB0202), the Minhang District Natural Science Research Project (grant no. 2017MHZ56) and the Shanghai Minhang District Center Hospital Grade Project (grant no. 2016MHJC10).

\section{Availability of data and materials}

The datasets used and/or analyzed in the present study are available from the corresponding author on reasonable request.

\section{Authors' contributions}

KS provided the conception and design of the study. ZX and CQ acquired the data. JW, YL and ML analyzed and interpreted the data and performed the statistical analysis. All authors read and approved the final manuscript.

\section{Ethics approval and consent to participate}

The use of animals was approved by the Animal Ethics Committee of Affiliated Hospital of Nantong University (Nantong, China).

\section{Patient consent for publication}

Not applicable.

\section{Competing interests}

The authors declare that they have no competing interests.

\section{References}

1. Santhakumar R, Ramalingam PK, Gayathri K, Manjunath BV, Karuppusamy N, Vetriveeran B, Selvamani S, Vishnuram P and Natarajan K: Pulmonary thromboembolism presenting as multiple pulmonary cavities. J Assoc Physicians India 64: 85-86, 2016.

2. Wood KE: Major pulmonary embolism: Review of a pathophysiologic approach to the golden hour of hemodynamically significant pulmonary embolism. Chest 121: 877-905, 2002.
3. Kostadima E and Zakynthinos E: Pulmonary embolism: Pathophysiology, diagnosis, treatment. Hellenic J Cardiol 48: 94-107, 2007.

4. Evans CE, Humphries J, Mattock K, Waltham M, Wadoodi A, Saha P, Modarai B, Maxwell PH and Smith A: Hypoxia and upregulation of hypoxia-inducible factor 1 \{alpha\} stimulate venous thrombus recanalization. Arterioscler Thromb Vasc Biol 30: 2443-2451, 2010.

5. Yilmaz M: Evaluation of Tp-e interval, Tp-e/qt ratio and Tp-e/Qtc ratio in patients with acute pulmonary thromboembolism. Am J Cardiol 121: e19, 2018.

6. Battistini B: Modulation and roles of the endothelins in the pathophysiology of pulmonary embolism. Canad J Physiol Pharmacol 81: 555-569, 2003

7. Kimura H, Okada O, Tanabe N, Tanaka Y, Terai M, Takiguchi Y, Masuda M, Nakajima N, Hiroshima K, Inadera H, et al: Plasma monocyte chemoattractant protein-1 and pulmonary vascular resistance in chronic thromboembolic pulmonary hypertension. Am J Respir Crit Care Med 164: 319-324, 2001.

8. Wan J, Lu LJ, Miao R, Liu J, Xu XX, Yang T, Hu QH, Wang J and Wang C: Alterations of bone marrow-derived endothelial progenitor cells following acute pulmonary embolism in mice. Exp Biol Med (Maywood) 235: 989-998, 2010.

9. Raskob GE, Angchaisuksiri P, Blanco AN, Buller $\mathrm{H}$, Gallus A, Hunt BJ, Hylek EM, Kakkar A, Konstantinides SV, McCumber M, et al: Thrombosis: A major contributor to global disease burden. Arterioscler Thromb Vasc Biol 34: 2363-2371, 2014.

10. Pieralli F, Olivotto I, Vanni S, Conti A, Camaiti A, Targioni G, Grifoni $S$ and Berni G: Usefulness of bedside testing for brain natriuretic peptide to identify right ventricular dysfunction and outcome in normotensive patients with acute pulmonary embolism. Am J Cardiol 97: 1386-1390, 2006.

11. Kostrubiec M, Pruszczyk P, Bochowicz A, Pacho R, Szulc M, Kaczynska A, Styczynski G, Kuch-Wocial A, Abramczyk P, Bartoszewicz Z, et al: Biomarker-based risk assessment model in acute pulmonary embolism. Eur Heart J 26: 2166-2172, 2005.

12. Babaoglu E, Hasanoglu HC, Senturk A, Karalezli A, Kilic H, Aykun G and Oztuna D: Importance of biomarkers in risk stratification of pulmonary thromboembolism patients. J Investig Med 62: 328-331, 2014.

13. Tang Z, Wang X, Huang J, Zhou X, Xie H, Zhu Q, Huang M and Ni S: Gene expression profiling of pulmonary artery in a rabbit model of pulmonary thromboembolism. PLoS One 11: e0164530, 2016.

14. He X, Zhang Z, Zhang Q and Yuan G: Selective recognition of $\mathrm{G}$-quadruplex in the vascular endothelial growth factor gene with small-molecule natural products by electrospray ionization (ESI) mass spectrometry and circular dichroism (CD) spectrometry. Canad J Chem 90: 55-59, 2012.

15. An R, Hagiya Y, Tamura A, Li S, Saito H, Tokushima D and Ishikawa T: Cellular phototoxicity evoked through the inhibition of human ABC transporter ABCG2 by cyclin-dependent kinase inhibitors in vitro. Pharm Res 26: 449-458, 2009.

16. Wang L, Cao C, Ma Q, Zeng Q, Wang H, Cheng Z, Zhu G, Qi J, Ma H, Nian H and Wang Y: RNA-seq analyses of multiple meristems of soybean: Novel and alternative transcripts, evolutionary and functional implications. BMC Plant Biol 14: 169, 2014

17. Szklarczyk D, Franceschini A, Kuhn M, Simonovic M, Roth A, Minguez P, Doerks T, Stark M, Muller J, Bork P, et al: The STRING database in 2011: Functional interaction networks of proteins, globally integrated and scored. Nucleic Acids Res 39 (Database Issue): D561-D568, 2011.

18. Shannon P, Markiel A, Ozier O, Baliga NS, Wang JT, Ramage D, Amin N, Schwikowski B and Ideker T: Cytoscape: A software environment for integrated models of biomolecular interaction networks. Genome Res 13: 2498-2504, 2003.

19. Bader GD and Hogue CW: An automated method for finding molecular complexes in large protein interaction networks. BMC Bioinformatics 4: 2, 2003

20. Ashburner M, Ball CA, Blake JA, Botstein D, Butler H, Cherry JM, Davis AP, Dolinski K, Dwight SS, Eppig JT, et al: Gene ontology: Tool for the unification of biology. The Gene Ontology Consortium. Nat Genet 25: 25-29, 2000.

21. Kanehisa M and Goto S: KEGG: Kyoto encyclopedia of genes and genomes. Nucleic Acids Res 28: 27-30, 2000.

22. Davis AP, Grondin CJ, Lennon-Hopkins K, Saraceni-Richards C, Sciaky D, King BL, Wiegers TC and Mattingly CJ: The comparative toxicogenomics database's 10th year anniversary: Update 2015. Nucleic Acids Res 43 (Database Issue): D914-D920, 2015. 
23. Yan $\mathrm{C}$, Wang $\mathrm{X}, \mathrm{Su} \mathrm{H}$ and Ying $\mathrm{K}$ : Recent progress in research on the pathogenesis of pulmonary thromboembolism: An old story with new perspectives. Biomed Res Int 2017: 6516791, 2017.

24. Wrobel JP, Thompson BR and Williams TJ: Mechanisms of pulmonary hypertension in chronic obstructive pulmonary disease: A pathophysiologic review. J Heart Lung Transplant 31: 557, 2012.

25. Jo JY, Lee MY, Lee JW, Rho BH and Choi WI: Leukocytes and systemic inflammatory response syndrome as prognostic factors in pulmonary embolism patients. BMC Pulm Med 13: 74, 2013.

26. Halici B, Sarinc US, Günay E, Nural S, Sen S, Akar O, Celik S and Unlu M: Assessment of inflammatory biomarkers and oxidative stress in pulmonary thromboembolism: Follow-up results. Inflammation 37: 1186-1190, 2014.

27. Marchena Yglesias PJ, Nieto Rodríguez JA, Serrano Martínez S, Belinchón Moya O, Cortés Carmona A, Díaz de Tuesta A, Bruscas Alijarde MJ and Ruiz Ribó MD: Acute-phase reactants and markers of inflammation in venous thromboembolic disease: Correlation with clinical and evolution parameters. An Med Interna 23: 105-110, 2006 (In Spanish).

28. Santosa F, Moerchel C, Berg C and Kröger K: Disproportional increase of pulmonary embolism in young females in Germany: Trends from 2005 to 2014 . J Thromb Thrombolysis 43: 417-422, 2017.

29. Bird ST, Delaney JA, Etminan M, Brophy JM and Hartzema AG: Drospirenone and non-fatal venous thromboembolism: Is there a risk difference by dosage of ethinyl-estradiol? J Thromb Haemost 11: 1059-1068, 2013.

30. Jiang X, Sung YK, Tian W, Qian J, Semenza GL and Nicolls MR: Graft microvascular disease in solid organ transplantation. J Mol Med (Berl) 92: 797-810, 2014.

31. Rodriguesdiez R, Gonzálezguerrero C, Ocañasalceda C, Rodriguesdiez RR, Egido J, Ortiz A, Ruizortega $M$ and Ramos AM: Calcineurin inhibitors cyclosporine $A$ and tacrolimus induce vascular inflammation and endothelial activation through TLR4 signaling. Sci Rep 6: 27915, 2016

32. Rafiee P, Johnson CP, Li MS, Ogawa H, Heidemann J, Fisher PJ, Lamirand TH, Otterson MF, Wilson KT and Binion DG: Cyclosporine a enhances leukocyte binding by human intestinal microvascular endothelial cells through inhibition of p38 MAPK and iNOS. Paradoxical proinflammatory effect on the microvascular endothelium. J Biol Chem 277: 35605-35615, 2002.
33. Chavez-Blanco A, Perez-Sanchez V, Gonzalez-Fierro A, Vela-Chavez T, Candelaria M, Cetina L, Vidal S and Dueñas-Gonzalez A: HER2 expression in cervical cancer as a potential therapeutic target. BMC Cancer 4: 59, 2004.

34. Balwierz AK: ERBB2 as a driver of an invasive phenotype of cells grown in 3D culture and an important regulator of oncogenic miRNAs' expression in breast cancer, 2017.

35. Ahmed AR: HER 2 expression is a strong independent predictor of nodal metastasis in breast cancer. J Egypt Natl Cancer Inst 28: 219-227, 2016.

36. Zhou X, De Luise C, Shen R, Bate A and Gatto N: Incidence of pulmonary embolism among postmenopausal (pm) women with $\mathrm{Er}^{+} / \mathrm{HER} 2^{-}$breast cancer. Pharmacoepidemiol Drug Safety 24: 372-373, 2015.

37. Massraro GD and Massaro D: Retinoic acid treatment abrogates elastase-induced pulmonary emphysema in rats. Nat Med 3: 675-677, 1997.

38. Jian-Lin XU, Fan XY, Guo YL, Jin MA and Wang WM: Effects of all-trans-retinoic acid on emphysema model in rats. Chin J New Drugs Clin Remed 29: 109-114, 2010 (In Chinese).

39. Faubert BL and Kaminski NE: AP-1 activity is negatively regulated by cannabinol through inhibition of its protein components, c-fos and c-jun. J Leukoc Biol 67: 259-266, 2000.

40. Eferl R, Ricci R, Kenner L, Zenz R, David JP, Rath M and Wagner EF: Liver tumor development. c-Jun antagonizes the proapoptotic activity of p53. Cell 112: 181-192, 2003.

41. Guo W, Pylayeva Y, Pepe A, Yoshioka T, Muller WJ, Inghirami G and Giancotti FG: Beta 4 integrin amplifies ErbB2 signaling to promote mammary tumorigenesis. Cell 126: 489-502, 2006.

This work is licensed under a Creative Commons Attribution-NonCommercial-NoDerivatives 4.0 International (CC BY-NC-ND 4.0) License. 